\title{
Nonlinear homotopy in geodesy
}

\author{
B. Paláncz ${ }^{1}$ J. L. Awange ${ }^{2}$
}

Received: 17 February 2016/Accepted: 12 March 2016/Published online: 30 March 2016

(C) Akadémiai Kiadó 2016

\begin{abstract}
In Paláncz et al. (J Geod 84: 79-85, 2009), linear homotopy was introduced and its applications to geodesy presented. Never before had the concept of nonlinear homotopy been used by the geodetic community. This is partly attributed to the complexity of the involved equations and partly due to the computational time required. Recently, however, Nor et al. (MATEMATIKA 29: 159-171, 2013) suggested the possibility of constructing nonlinear homotopy. In this short note, Nor et al. (MATEMATIKA 29: 159-171, 2013) idea is developed for geodetic applications and an example of its use illustrated.
\end{abstract}

Keywords Nonlinear homotopy · GPS · Geodesy

\section{Nonlinear Homotopy}

Since the linear homotopy function $H(\mathrm{x}, \lambda)$ is just a linear combination of the starting $p(\mathrm{x})$ and the target system $q(\mathrm{x})$, it is a first order polynomial of the homotopy parameter $\lambda$ (see, e.g., Awange et al. 2010, p. 64) given by

$$
H(x, \lambda)=(1-\lambda) p(x)+\lambda q(x) .
$$

\footnotetext{
J. L. Awange

j.awange@curtin.edu.au

B. Paláncz

palancz@epito.bme.hu
}

1 Department of Photogrammetry and Geoinformatics, Budapest University of Technology and Economics, Budapest 1521, Hungary

2 Western Australian Centre for Geodesy and The Institute for Geoscience Research, Curtin University, Perth, Australia 
It would be reasonable to think that a nonlinear homotopy, i.e.,- - let us say a second order homotopy-can be constructed as a second order polynomial of $\lambda$. However, Nor et al. (2013) suggested the following analogy to create a second order homotopy function. Nor et al. (2013) idea of nonlinear homotopy function comes from the construction of the Bezier splines. Bezier curves are used to draw smooth curves along points on a path. In case of two points $\left(P_{0}, P_{1}\right)$ in Fig. 1 , the point $Q_{0}$ runs from $P_{0}$ to $P_{1}$ while the parameter $\lambda$ changes from 0 to 1 , i.e.,

$$
\overrightarrow{Q_{0}}=(1-\lambda) \overrightarrow{P_{0}}+\lambda \overrightarrow{P_{1}}, \quad \lambda \in[0,1]
$$

In case of three points $\left(P_{0}, P_{1}, P_{2}\right)$, e.g., Fig. 2 , the point $Q_{0}$ runs from $P_{0}$ to $P_{1}$, while point $Q_{1}$ runs from $P_{1}$ to $P_{2}$, i.e.,

$$
\overrightarrow{Q_{1}}=(1-\lambda) \overrightarrow{P_{1}}+\lambda \overrightarrow{P_{2}}, \quad \lambda \in[0,1]
$$

and point $R_{0}$ runs along a smooth path from $P_{0}$ to $P_{2}$, i.e.,

$$
\overrightarrow{R_{0}}=(1-\lambda) \overrightarrow{Q_{0}}+\lambda \overrightarrow{Q_{1}}, \quad \lambda \in[0,1],
$$

or substituting Eqs. (2) and (3) in (4) leads to

$$
\overrightarrow{R_{0}}=(1-\lambda)^{2} \overrightarrow{P_{0}}+2(1-\lambda) \lambda \overrightarrow{P_{1}}+\lambda^{2} \overrightarrow{P_{2}}, \quad \lambda \in[0,1] .
$$

Considering the analogy between the Bezier curve construction and the quadratic homotopy function, Nor et al. (2013) suggested the following casting,

$$
\begin{gathered}
P_{0} \sim p(x) \\
P_{1} \sim H(x, \lambda) \\
P_{2} \sim q(x),
\end{gathered}
$$

from which the second order homotopy function becomes

Fig. 1 Linear Bezier spline

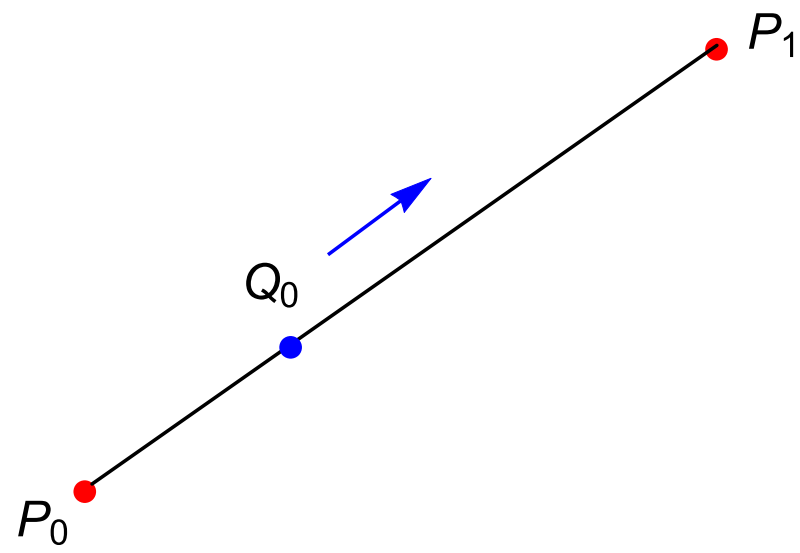




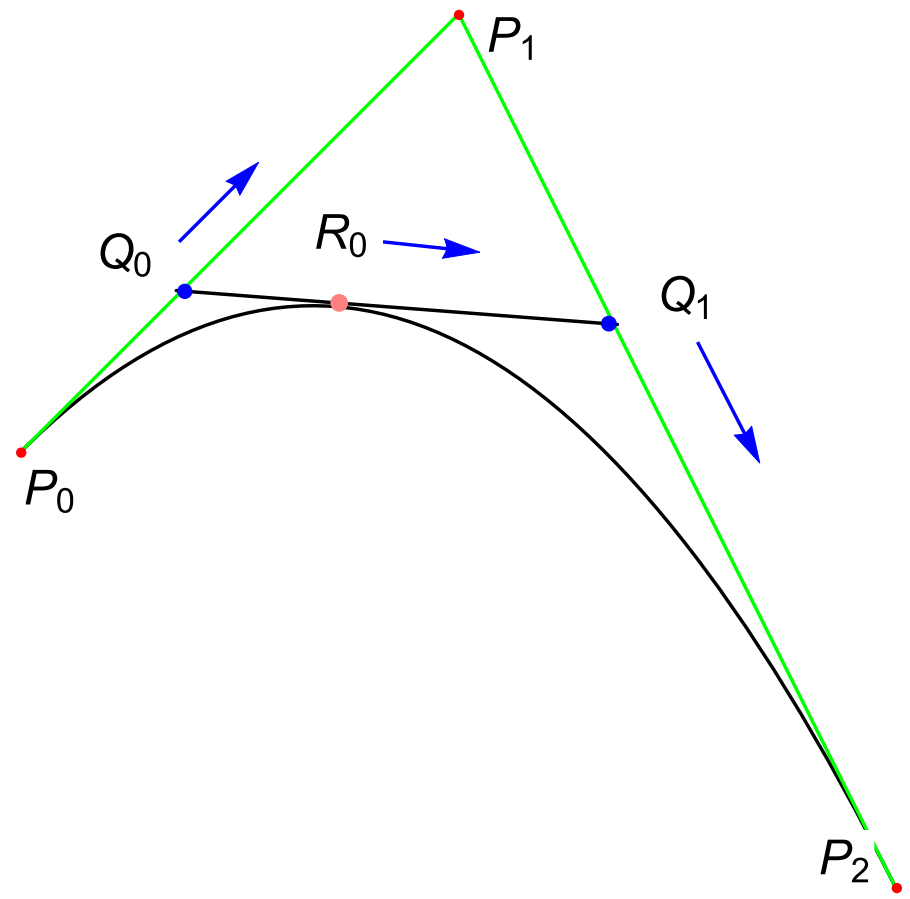

Fig. 2 Quadratic Bezier spline

$$
H_{2}(x, \lambda)=(1-\lambda)^{2} p(x)+2 \lambda(1-\lambda) H(x, \lambda)+\lambda^{2} q(x) .
$$

Substituting $H(x, \lambda)$ from Eq. (1) in (6) leads to

$$
(1-\lambda)^{2} p(x)+2 \lambda(1-\lambda)((1-\lambda) p(x)+\lambda q(x))+\lambda^{2} q(x),
$$

which surprisingly is a third order polynomial of $\lambda$.

\section{Example of its Application to Geodesy (GPS Pseudorange Problem)}

In order to discover the features of the nonlinear homotopy (Eq. 7), the overdetermine GPS positioning problem (i.e., the case of $n>4$ satellite) is illustrated. The prototype equation expressing the known satellite positions $\left(a_{i}, b_{i}, c_{i}\right)$, unknown receiver position $\left(x_{1}, x_{2}, x_{3}\right)$, and clock bias $\left(x_{4}\right)$ is (Awange and Paláncz 2016):

$$
g_{i}=d_{i}-\left(\sqrt{\left(x_{1}-a_{i}\right)^{2}+\left(x_{2}-b_{i}\right)^{2}+\left(x_{3}-c_{i}\right)^{2}}-x_{4}\right), \quad \text { for } i=1,2, \ldots n .
$$

The overdetermined system can then be converted into a determined system using the least square technique. Given the objective function to be minimized as $G\left(x_{1}, x_{2}, x_{3}, x_{4}\right)=$ $\sum_{i=1}^{n} g_{i}^{2}$, its partial derivative with respect to the unknown receiver position and clock bias, variables $x_{i} \mid \mathrm{i}=1,2, \ldots 4$ leads to a determined nonlinear system of four equations 
Table 1 Results of the solutions of the GPS pseudorange problem from various methods.

\begin{tabular}{lll}
\hline Method & Running time (s) & Norm of error \\
\hline Linear homotopy (1) & 1.30 & 0.04424 \\
Nonlinear homotopy & 1.94 & 0.03125 \\
Numerical Groebner basis & 0.06 & 0.0690 \\
Linear homotopy (2) & 2.58 & 0.0358 \\
\hline
\end{tabular}

$$
\begin{gathered}
g_{1}=\sum_{i=0}^{-1+n}-\frac{2\left(x_{1}-a_{i}\right)\left(-x_{4}-\sqrt{\left(x_{1}-a_{i}\right)^{2}+\left(x_{2}-b_{i}\right)^{2}+\left(x_{3}-c_{i}\right)^{2}}+d_{i}\right)}{\sqrt{\left(x_{1}-a_{i}\right)^{2}+\left(x_{2}-b_{i}\right)^{2}+\left(x_{3}-c_{i}\right)^{2}}} \\
g_{2}=\sum_{i=0}^{-1+n}-\frac{2\left(x_{2}-b_{i}\right)\left(-x_{4}-\sqrt{\left(x_{1}-a_{i}\right)^{2}+\left(x_{2}-b_{i}\right)^{2}+\left(x_{3}-c_{i}\right)^{2}}+d_{i}\right)}{\sqrt{\left(x_{1}-a_{i}\right)^{2}+\left(x_{2}-b_{i}\right)^{2}+\left(x_{3}-c_{i}\right)^{2}}} \\
g_{3}=\sum_{i=0}^{-1+n}-\frac{2\left(x_{3}-c_{i}\right)\left(-x_{4}-\sqrt{\left(x_{1}-a_{i}\right)^{2}+\left(x_{2}-b_{i}\right)^{2}+\left(x_{3}-c_{i}\right)^{2}}+d_{i}\right)}{\sqrt{\left(x_{1}\right)^{2}+\left(x_{2}-b_{i}\right)^{2}+\left(x_{3}-c_{i}\right)^{2}}} \\
g_{4}=\sum_{i=0}^{-1+n}-2\left(-x_{4}-\sqrt{\left(x_{1}-a_{i}\right)^{2}+\left(x_{2}-b_{i}\right)^{2}+\left(x_{3}-c_{i}\right)^{2}}+d_{i}\right) .
\end{gathered}
$$

The resulting determined system above is then solved using both linear and nonlinear second order homotopy. The starting system can be created via fixed point homotopy (see, e.g., Paláncz et al. 2009). First, computations are made with the same medium step size for both linear as well as nonlinear homotopy. The results of the computations are summarized in Table 1 where they show the running time of the nonlinear homotopy to be higher than that of the linear homotopy, but with smaller error. To clarify the situation, a second computation with linear homotopy (2) using smaller step size in order to decrease its error was undertaken. In that case, the running time increased considerably and the error was higher than that of the nonlinear homotopy. It means that nonlinear homotopy may provide shorter running time at the same error limit. As a check to the computation, the system of nonlinear equations is solved using numerical Groebner basis. Groebner basis leads to a faster computational time but with higher error. Considering its running time, one should take into consideration that numerical Groebner basis is a compiled function, while the homotopy function runs in interpreter mode.

\section{References}

Awange JL, Paláncz B (2016) Geosptatial algebraic computations. In: Theory and applications, 3rd edn. Springer, Berlin

Awange JL, Grafarend EW, Palancz B, Zaletnyik P (2010) Algebraic geodesy and geoinformatics. Springer, Berlin

Nor HM, Ismail AM, Majid AA (2013) Quadratic bezier homotopy function for solving system of polynomial equations. MATEMATIKA 29(2):159-171

Paláncz B, Awange JL, Zaletnyik P, Lewis RH (2009) Linear homotopy solution of nonlinear systems of equations in geodesy. J Geod 84(1):79-95 